\title{
Individualized Postpartum Hemorrhage Management by the ROTEM Guidance, Illuminating the Shadow Zone of the Treatment
} \\ "Corresponding author: Department of Anesthesiology, Sarem Hospital, Tehran, Iran. Email: a-behseresht@razi.tums.ac.ir
}

Received 2021 March 31; Accepted 2021 April 11.

Keywords: Fibrinogen, Point of Care Testing, Postpartum Hemorrhage, ROTEM

\section{Dear Editor,}

Primary postpartum hemorrhage is defined as maternal hemorrhage within the first 24 hours after delivery, which is the most significant cause of maternal mortality. In these cases, surgical and medical treatments should be considered based on the mother's condition. Failure to quickly take bleeding under control is highly dangerous and often ends in disseminated intravascular coagulation.

Most coagulation factors, including fibrinogen, physiologically increase during pregnancy, which enables almost all mothers to physiologically compensate for the regular delivery bleeding without the need for any specific treatment (1). Fibrinogen consumption plays a major role in peripartum hemorrhage as this phenomenon is fully seen in placental abruption. Blood fibrinogen levels less than $200 \mathrm{mg} / \mathrm{dl}$ at the time of maternal hemorrhage are directly related to hemorrhage severity. Generally, at a blood fibrinogen level of higher than $400 \mathrm{mg} / \mathrm{dl}$, severe hemorrhage is improbable. Therefore, recent studies have recommended that maternal blood fibrinogen levels should be maintained at least $200 \mathrm{mg} / \mathrm{dL}$, and its decline should be prevented (2).

Persistent postpartum hemorrhage is usually triggered by hidden coagulopathies. On the other hand, most routine coagulation tests (platelet count, plasma fibrinogen level, PT, PTT, and INR) take at least 60 minutes to be completed. Consequently, it is quite common that the patient's coagulation status will change entirely at the time of receiving the tests' results. Hence, a rapid test at the patient's bedside is required to obtain accurate information about the patient's continuously fluctuating coagulation status (3). Thromboelastometry, as a quick and reliable method for coagulation assessment at the patient's bedside, has been recently employed in similar situations like severe trauma, cardiothoracic surgery, and liver transplantation, in which monitoring the coagulation status of patients plays a crucial role in determining their outcomes. Likewise, this method can help to manage postpartum hemorrhage as well. The main advantage of rotational thromboelastometry (ROTEM) is the possibility of the real-time evaluation of homeostasis, which offers patients to receive individualized therapy. The test is performed using a whole blood sample (4). Early preemptive treatments can largely prevent the progression of postpartum hemorrhage to DIC and the incidence of lethal treatment complications like transfusion-related circulatory overload (TACO) and transfusion-related acute lung injury (TRALI) afterward.

Due to relatively high plasma fibrinogen levels in term pregnancies (400 - $600 \mathrm{mg} / \mathrm{dL}$ ), recent studies have presented thresholds for preventing and treating postpartum hemorrhage based on the ROTEM output data.

It seems that early ROTEM testing and the early administration of fibrinogen concentrates in patients with the FIBTEM A5 of $<12 \mathrm{~mm}$ may improve their outcomes. Meanwhile, an EXTEM clotting time (CT) of $>100$ seconds is also assumed to be noteworthy, and administering coagulation factors' concentrates seems to be helpful in such cases. At the end of every treatment stage, coagulation alterations are monitored by repeating the ROTEM test in short intervals, and additional treatments are administered if needed (5). Although more investigations are needed in this era, it seems that postpartum hemorrhage management is becoming more precise using the ROTEM as a point of care test, delivering better outcomes. It is recommended for anesthesiologists, as the head of the resuscitation team, to 
boost their knowledge about this point of care test.

\section{Footnotes}

Authors' Contribution: E.B. and S.S.S. developed the original idea and the protocol, analyzed and interpreted the data, wrote the manuscript. S. S. and A.B. contributed to the development of the protocol, data analysis, manuscript preparation.

Conflict of Interests: There is no conflict of interest.

Funding/Support: There is no funding/support.

\section{References}

1. O’Brien KL, Shainker SA, Lockhart EL. Transfusion management of obstetric hemorrhage. Transfus Med Rev. 2018;32(4):249-55. doi: 10.1016/j.tmrv.2018.05.003. [PubMed: 29934136].
2. Collins PW, Cannings-John R, Bruynseels D, Mallaiah S, Dick J, Elton $\mathrm{C}$, et al. Viscoelastometric-guided early fibrinogen concentrate replacement during postpartum haemorrhage: OBS2, a doubleblind randomized controlled trial. BrJAnaesth. 2017;119(3):411-21. doi: 10.1093/bja/aex181. [PubMed: 28969312].

3. Walsh M, Thomas S, Kwaan H, Aversa J, Anderson S, Sundararajan $\mathrm{R}$, et al. Modern methods for monitoring hemorrhagic resuscitation in the United States: Why the delay? J Trauma Acute Care Surg. 2020;89(6):1018-22. doi: 10.1097/TA.0000000000002977. [PubMed: 33048910].

4. Smith AR, Karim SA, Reif RR, Beck WC, Taylor JR, Davis BL, et al. ROTEM as a predictor of mortality in patients with severe trauma. J Surg Res. 2020;251:107-11. doi:10.1016/j.jss.2020.01.013. [PubMed: 32114212].

5. Lee J, Eley VA, Wyssusek KH, Coonan E, Way M, Cohen J, et al. Baseline parameters for rotational thromboelastometry (ROTEM(R)) in healthy women undergoing elective caesarean delivery: A prospective observational study in Australia. Int J Obstet Anesth. 2019;38:10-8. doi: 10.1016/j.ijoa.2019.01.008. [PubMed:30770209]. 